\title{
Corela
}

Cognition, représentation, langage

HS-1 | 2005

Colloque AFLS

\section{Un recensement classé des constructions verbales : méthode et perspectives}

\section{Stéphanie Giron}

\section{(2) OpenEdition}

\section{Journals}

Édition électronique

URL : http://journals.openedition.org/corela/1140

DOI : $10.4000 /$ corela. 1140

ISSN : 1638-573X

\section{Éditeur}

Cercle linguistique du Centre et de I'Ouest - CerLICO

\section{Référence électronique}

Stéphanie Giron, «Un recensement classé des constructions verbales : méthode et perspectives », Corela [En ligne], HS-1 | 2005, mis en ligne le 16 février 2005, consulté le 01 mai 2019. URL : http:// journals.openedition.org/corela/1140 ; DOI : 10.4000/corela.1140

Ce document a été généré automatiquement le 1 mai 2019.

\section{(c) (i) (2)(2)}

Corela - cognition, représentation, langage est mis à disposition selon les termes de la licence Creative Commons Attribution - Pas d'Utilisation Commerciale - Partage dans les Mêmes Conditions 4.0 International. 


\title{
Un recensement classé des constructions verbales : méthode et perspectives
}

\author{
Stéphanie Giron
}

1 La constitution d'un corpus n'est pas chose évidente ; c'est la raison pour laquelle donner ici une idée de la méthode de travail nous semblait pertinent. Ainsi, depuis les enquêtes de terrain jusqu'à la transcription des enregistrements, nous présenterons, dans un premier temps, la méthode que nous avons utilisée pour constituer ce corpus et les étapes chronologiques importantes de sa constitution.

2 D'autre part, ce corpus nous sert de base à un recensement et à un classement des constructions verbales (désormais $\mathrm{CV}$ ) dans le cadre de notre thèse (en cours). Après avoir présenté le corpus, nous expliquerons donc la méthode utilisée pour le recensement et pour le classement de ces $\mathrm{CV}$, en prenant pour exemple l'étude du verbe aller. In fine, nous indiquerons quelques perspectives de recherches qui pourraient s'avérer intéressantes pour poursuivre et utiliser ce travail.

\section{Présentation du corpus ${ }^{1}$}

3 Nous avons constitué durant l'année 2001 un corpus d'environ 100000 mots, à partir d'enquêtes effectuées à Montluçon, Vichy et Moulins, auprès de 9 locuteurs dans chacune de ces villes (les critères de sélection de ces locuteurs seront précisés dans la partie consacrée aux enquêtes).

4 La durée de chaque enregistrement est de 30 minutes, dont 15 sont transcrites, selon les conventions données par l'équipe DELIC ${ }^{2}$. Ces enregistrements sont donc transcrits et informatisés (son et texte sont disponibles sur CD-ROM), voici leurs références :

\begin{tabular}{|l|l|l|l|}
\hline & MONTLUCON & MOULINS & VICHY \\
\hline
\end{tabular}




\begin{tabular}{|l|l|l|l|}
\hline 18-30 ans niveau collège & CHOCM 10 & COMMO 14 & HOTEV 26 \\
\hline $18-30$ ans niveau bac & MUMON 44 & FANMO 16 & JOLIEV 32 \\
\hline $18-30$ ans niveau bac+3 & SANDM $\sim 8$ & FRUIT 22 & MARCH $\sim 40$ \\
\hline $30-65$ ans niveau collège & MADMO 34 & COIFM 12 & MONOV 42 \\
\hline $30-65$ ans niveau bac & PAPMO 50 & GILMO 24 & QUALV 56 \\
\hline $30-65$ ans niveau bac+3 & MUSIK 46 & FRANM $~ 20$ & JAMES 30 \\
\hline+65 ans niveau collège & MAMMO 38 & MALLM 36 & BIJOU 6 \\
\hline+65 ans niveau bac & FRAMO 18 & INSTI 28 & NINAV 48 \\
\hline+65 ans niveau bac+3 & BRUNMO 8 & PHYMO 54 & PETIV 52 \\
\hline
\end{tabular}

5 Tous ces enregistrements sont de type "parole privée ", c'est-à-dire qu'ils revêtent la forme d'un entretien entre le locuteur interrogé et l'enquêteur (le temps de parole étant occupé à environ $95 \%$ par le locuteur interrogé).

6 Les 9 corpus de Montluçon n'ont pas de thème bien défini : il s'agissait souvent pour le locuteur, par exemple de nous raconter un fait marquant de sa vie; en revanche, les corpus de Vichy et de Moulins ont été réalisés à partir du thème " petit commerce ».

7 Nous utilisons fréquemment les notions de corpus et de sous-corpus. Cependant, le terme sous-corpus risquera d'être ambigu, dans la mesure où il peut à la fois référer :

- au premier niveau ${ }^{3}$, que nous posons comme le plus « précis », c'est-à-dire celui à l'intérieur duquel on dénombre 27 sous-corpus, soit un par locuteur.

- au deuxième niveau, que nous posons comme le moins "précis ", dans la mesure où les variables qui le composent, basées sur les critères de sélection des locuteurs (3 villes, 3 tranches d'âge, 3 niveaux d'études) multiplient de manière très importante les possibilités combinatoires de recherche. On pourra ainsi sous-catégoriser en fonction de la ville (3 souscorpus : Montluçon, Vichy, Moulins), en fonction de l'âge (3 sous-corpus : 18-30 ans, 30-65 ans, +65 ans) ou en fonction du niveau d'étude (3 autres sous-corpus : collège, bac ou bac +3 ). Mais on pourra aussi sous-catégoriser en associant les différentes variables : les locuteurs de tel âge, de tel niveau d'étude, de telle ville, ce qui donne $3^{3}$, c'est-à-dire 27 souscatégorisations potentielles.

8 Après cette présentation très générale de notre corpus, nous allons à présent détailler la méthode que nous avons utilisée pour effectuer les enquêtes, tout en précisant lorsqu'il le sera nécessaire les points qui nous ont paru délicats, ou ceux dont la réalisation s'avère imparfaite.

\section{Enquêtes et transcriptions}

Plusieurs paramètres doivent être pris en compte lors de la constitution d'un corpus : en ce qui concerne l'enquête de terrain, le choix des locuteurs enregistrés est important si l'on veut rendre compte le plus finement possible de l'état d'une langue à un moment donné de son histoire, et les critères de sélection qui précèdent le choix des personnes 
interrogées permettent d'introduire diverses variables au cours de l'étude (variable géographique, niveau d'étude, et âge), qui n'ont pas été utilisées au cours de ce travail, mais qui pourraient se révéler intéressantes pour des recherches dans d'autres domaines que celui de la syntaxe, par exemple la sociolinguistique urbaine.

Les enquêtes ont été réalisées en partie suivant les indications données par l'équipe DELIC. Lors de l'élaboration du corpus Français de Référence, il s'agissait pour elle d'enregistrer des locuteurs selon leur âge, réparti en 3 tranches, leur niveau d'étude réparti également en 3 niveaux, et le type de parole enregistrée (3 types de parole: privée, publique et professionnelle). Nous avons repris les deux premiers critères, l'âge et le niveau d'étude, mais n'avons pas enregistré plusieurs types de parole: nous n'avons que des "paroles privées». Cependant, nous avons ajouté à cet échantillonnage un critère géographique, qui apparait dans le Français de Référence, mais de manière moins équilibrée (27 corpus pour Paris et sa banlieue, 49 pour le nord de la France et 55 pour le sud). En effet, nous avons enregistré 9 locuteurs dans chacune des trois villes de l'Allier, ce qui fournit à ce corpus un équilibre pouvant permettre, pour des recherches futures, une étude de la variation linguistique dans ce département, qui est situé au cœur de ce que les dialectologues nomment le croissant, c'est-à-dire la zone où passe la frontière entre langue d'oïl et langue d'oc, et où l'on trouve, au sud-est, un peu de francoprovençal.

Lorsqu'il y a enregistrement de discours, plusieurs méthodes sont possibles, selon l'étude que l'on veut mener à bien, car "les différents types de corpus de langue parlée se définissent généralement par les objectifs qu'ils se donnent: faciliter l'enseignement, faire de la recherche linguistique, collaborer à de plus larges recherches statistiques, participer à des recherches de sociolinguistique, etc. » (C. Blanche-Benveniste, in Bilger, 2000 : p. 14). Notre étude portant sur la syntaxe, l'intérêt est de laisser libre cours à la parole des locuteurs, afin de pouvoir observer les unités de discours et plus précisément les constructions verbales que nous observerons dans la deuxième partie de ce travail.

Notre enquête est donc «libre ", si ce n'est qu'un thème est défini auparavant, ce qui permet au locuteur de parler relativement facilement. Nous entendons par « libre » le fait que l'entretien n'est pas directif: nous n'avions pas élaboré de questionnaire comme l'avaient fait les enquêteurs travaillant sur le Corpus d'Orléans (Biggs et Blanc, 1971). Pendant une demie-heure, le locuteur parle d'un sujet défini au préalable de l'enregistrement. Comme nous le verrons lors de cette partie, « la spontanéité de la prise de parole, qui est un facteur intéressant pour l'analyse des interactions, n'est pas toujours un bon atout pour l'observation grammaticale. Les conversations à bâtons rompus, qui représentent assez bien les situations de parole spontanées, ne livrent généralement qu'une syntaxe limitée, exploitant des schémas répétitifs et souvent interrompus » (C. Blanche-Benveniste, in Bilger, 2000 : p. 20). C'est pourquoi nous sommes intervenue le moins possible dans le discours du locuteur : nous le relancions parfois en posant une question ouverte, ce qui permettait de développer un point précis ou de relancer son discours lorsqu'il y avait essoufflement et que, manifestement, le locuteur était «à court » d'idées.

D'autres paramètres se révèlent importants lors de l'enregistrement : le lieu, la position des locuteurs, l'intervention ou non de l'enquêteur, etc. Nous en présenterons quelquesuns au troisième point de cette partie. Auparavant, observons dans le détail les critères retenus pour la sélection des locuteurs ainsi que le matériel que nous avons utilisé. 


\section{Méthode retenue pour les enquêtes}

14 Le recueil des données est une étape importante de la constitution d'un corpus : d'une part, le travail est très long, qu'il s'agisse des enquêtes de terrain ou de la transcription, et d'autre part, la qualité de l'analyse dépend essentiellement de celle du corpus; c'est pourquoi il nous semble pertinent de proposer une méthode détaillée du travail d'enquête, qu'il s'agisse des critères de sélection des locuteurs ou des problèmes dus, dans notre corpus, à la cohérence thématique ou ceux inhérents à tout enregistrement de discours.

Cette partie montrera donc, chronologiquement, les différentes étapes de la constitution d'un corpus : dans un premier temps, la définition des critères précédant le choix des locuteurs, puis le matériel utilisé, la nature de l'entretien et enfin, les conditions d'enregistrement.

\subsection{Choix des critères de sélection des locuteurs}

Pour être pertinemment exploitable à la fois en syntaxe, mais aussi dans d'autres domaines de la linguistique, comme, par exemple, la sociolinguistique urbaine, le choix des locuteurs doit être opéré en fonction de plusieurs critères ; dans notre cas, nous avons utilisé les critères d'enquêtes utilisés par l'équipe DELIC lors de l'élaboration du Corpus français parlé de référence, au nombre de trois : critère géographique (3 zones urbaines de l'Allier, Vichy, Montluçon et Moulins), critère socioprofessionnel (3 niveaux d'études: collège, bac et bac + 3), critère d'âge (3 tranches d'âge : 18-30 ans, 30-65 ans et + de 65 ans). Ces trois critères peuvent servir de variables dans une étude ultérieure, puisque la variation linguistique peut être analysée d'un point de vue géographique, d'un point de vue sociolinguistique ou encore diachronique (l'hypothèse étant qu'un locuteur de + de 65 ans n'utilisera pas nécessairement les mêmes tournures syntaxiques qu'un locuteur de 18 ans, et que, même si l'on sait que la syntaxe n'évolue pas aussi rapidement que le lexique, des tournures sont délaissées au profit d'autres, etc.).

\subsubsection{Critère géographique}

Le critère géographique est celui favorisé par les dialectologues. Il permet d'observer les variations linguistiques potentielles d'un lieu à un autre si l'on choisit plusieurs lieux d'enquêtes hétérogènes (Nord/Sud, par exemple), ou, au contraire, il permet d'annuler cette variable si l'on choisit des lieux d'enquêtes situés dans une même région, dans un même département ou dans une même ville. Comme le note F. Gadet (1971 : p. 75) dans un de ses articles consacré aux variations sociales de la langue :

L'étude systématique des différences qu'elles [les variations géographiques] déterminent est du ressort de la dialectologie. Elle ne nous intéresse ici que dans la mesure où nous voyons la nécessité de choisir des sujets de même origine régionale, pour éviter l'interférence des variations linguistiques attribuables à des causes sociologiques avec celles dues à des variétés régionales.

Cependant, il est important de noter que le lieu de notre enquête peut être considéré comme non homogène, du fait de sa situation géolinguistique : le département de l'Allier, situé en partie dans la zone que les dialectologues nomme le croissant, est à la frontière 
entre langue d'oïl, langue d'oc et francoprovençal. Les enquêtes étant menées en zones urbaines, nous pensons avoir limité ainsi les influences dialectales, tant il est vrai que

[un des] principaux facteurs pouvant avoir une répercussion sur la façon de parler [est] la localisation de l'habitat à l'intérieur d'une même région (ville ou campagne). (ibid)

Ainsi, le choix d'effectuer les enquêtes en zones urbaines permet d'unifier ce facteur et par conséquent de limiter l'importance des variations géolinguistiques que l'on aurait eues si les enquêtes avaient été menées à la campagne. Mais leur étude peut se révéler intéressante. En effet, l'emplacement géographique des trois villes à l'intérieur du département peut permettre de comparer le français urbain standard parlé dans la zone d'oïl (Moulins) et dans la zone médiane entre oïl et oc, située dans le croissant (Montluçon au nord et Vichy au sud). Ainsi, ce corpus peut permettre de vérifier l'influence dialectale sur le parler standard, ce que nous ne ferons pas dans le cadre de notre étude, car nous n'utiliserons pas ce critère; mais, comme nous l'avons mentionné plus haut, plus un corpus est composé de sous-types (qui sont délimités par les différentes variables retenues), plus son exploitation peut se révéler riche pour des études ultérieures.

\subsubsection{Critère « niveau d'étude »}

Ce critère comprend trois différents niveaux d'études :

- collège

- baccalauréat

- baccalauréat +3

Il faut cependant préciser que ces trois niveaux sont indicatifs : un individu scolarisé jusqu'à la terminale et qui n'a pas passé l'épreuve du baccalauréat entre dans la catégorie "baccalauréat»; en revanche, un individu scolarisé jusqu'en seconde entre dans la catégorie "collège "; un individu ayant fait plus de trois années d'études supérieures entre dans la catégorie « baccalauréat +3 ».

Selon F. Gadet (1971: p. 75), le niveau d'étude est un des trois principaux indices d'appartenance d'un individu à une classe sociale, avec le salaire et la profession, qui, selon elle, doivent « être étudiés en bloc, sans perdre de vue qu l'un de ces facteurs peut être prépondérant ». Ne retenir que le niveau d'étude pourra donc apparaître réducteur à certains sociolinguistes; cependant, la profession de chaque locuteur apparaît dans la fiche signalétique qui lui correspond et qui figure en annexe. Ainsi, seul le salaire du locuteur n'apparaît pas.

Ce critère est intéressant pour plusieurs raisons: d'une part, il permet d'éviter de demander au locuteur (qu'on voit souvent pour la première fois) quels sont ses revenus, question qui peut bien sûr être très gênante pour l'« enquêté ", mais aussi pour l'enquêteur ; d'autre part, même si la scolarisation n'est pas le seul moyen éducatif ni le seul lieu d'apprentissage de la langue, le niveau d'étude paraît pertinent pour rendre compte de différents niveaux socioprofessionnels.

Passons maintenant à la description de ce que nous appelons « critère âge ».

\subsubsection{Critère « âge »}

Ce critère comprend trois tranches d'âge :

- $18-30$ ans 
- 30-65 ans

- + de 65 ans

avoir une vision assez précise de l'évolution d'une langue au cours du temps. En effet, un locuteur de 70 ans emploiera quelques tournures (et parfois quelques mots issus du patois) que n'emploierait pas un autre locuteur de 18 ou de 40 ans (notamment le passé surcomposé, employé par les locuteurs de plus de 65 ans, mais très rarement par les autres), comme F. Gadet le souligne (ibid.) :

$\mathrm{Du}$ fait qu'une langue est perpétuellement en mouvement, il arrive constamment que certains traits soient réalisés différemment chez les jeunes et chez les vieux. Par exemple en français, il y a une disparition lente, mais progressive, de l'opposition entre /a/ et $/ \alpha /$, entre / $\tilde{\varepsilon} /$ et / $\tilde{e} /$, entre le /e/ du futur et le / $/ \varepsilon / d u$ conditionnel, chez les jeunes. Au niveau lexicologique, notons un emploi plus systématique de termes argotiques dans la jeunesse.

Nous venons de passer en revue les trois critères de sélection que nous avons retenus pour nos enquêtes. Une fois cette étape achevée, l'enquêteur doit trouver les personnes y correspondant. La tâche se révèle plus ou moins difficile selon qu'il connaît ou non la localité sur laquelle il travaille. Elle peut l'être aussi à cause de tout autre phénomène, que nous n'étudierons pas ici, et qui peuvent être aussi bien liés à la timidité de l'enquêteur, à sa capacité d'adaptation sociale, ou encore à la relation même qu'il entretient avec le locuteur qu'il interroge.

\subsection{Matériel utilisé}

Nous avons utilisé, lors de nos enquêtes, le matériel suivant :

- un enregistreur mini-disque Sony MZ-R700

- un microphone de table Sony ECM-MS907

- un microphone cravate VIVANCO EM116 (utilisé lors des enquêtes dans les petits commerces, où les personnes interrogées pouvaient continuer à travailler sans être trop gênées par l'enquêteur).

Par la suite, nous avons copié les enregistrements effectués sur des mini-disques sur des $\mathrm{CD}$ de données au format mp3 à l'aide d'un logiciel gratuit disponible sur Internet ${ }^{4}$ (Electronic Cosmo's MPEG suite), et ajouté à cela les transcriptions au format Word20005. Les fichiers sons et textes étant référencés de manière identique, la consultation se révèle ainsi plus simple, par exemple: les corpus BIJOUV 1.doc et BIJOUV 1.mp3 sont, respectivement, la version transcrite et la version son du même enregistrement.

\subsection{Enquête non directive}

31 Contrairement à la plupart des enquêtes sociolinguistiques (notamment le Corpus d'Orléans), nous n'avons pas utilisé de questionnaire, mais avons laissé libre cours à la parole du locuteur, ce que les sociologues nomment entretien non-directif.

32 Jacqueline FREYSSINET-DOMINJON (1997 : pp. 150-151) définit ainsi l'entretien non directif : 
L'entretien non directif, également qualifié d'entretien en profondeur, se place au plus faible degré de structuration de l'échange verbal et au plus haut degré de liberté de parole de la personne enquêtée. L'enquêteur ayant posé au départ le thème de la recherche, adopte [une] attitude [...] faite d'écoute empathique et de neutralité congruente. [...]

L'usage de ce type d'entretien [...] est recommandé [...] toutes les fois que la recherche porte sur des représentations souvent latentes plutôt que sur des faits précis.

\section{décrire un ensemble de textes, il est nécessaire que les propriétés constitutives de chaque} production orale soient en partie semblables. Nous disons en partie car même si, théoriquement, les conditions d'enregistrement doivent être identiques pour chaque locuteur, nous avons eu quelques difficultés d'application pratique, dans les cas notamment où nous enregistrions des locuteurs sur leur lieu de travail. Ceci est sans doute un des reproches qui pourront nous être adressés, nous en avons bien conscience.

Concernant notre corpus, voici les conditions d'enregistrement :

\subsubsection{Lieu d'enregistrement}

Lorsque l'enregistrement se déroule chez l'enquêté, ce dernier propose généralement la pièce habituelle de réception la plus calme (cuisine, bureau ou jardin). 24 corpus sont enregistrés ainsi.

Lorsque l'enregistrement ne se déroule pas chez l'enquêté, il est effectué soit sur son lieu de travail à l'aide d'un micro-cravate pour ne pas gêner l'accomplissement de certaines tâches ( 2 corpus enregistrés ainsi : James 30 et Monov 42), soit à la terrasse d'un café en zone piétonne avec le microphone « traditionnel » (Mumon 44). 


\subsubsection{Position enquêteur / enquêté} de sélection de l'échantillon de locuteurs jusqu'au matériel utilisé et aux conditions d'enregistrement. Après cela commence un long travail d'écoute et de transcription, sur lequel nous ne reviendrons pas ici, cette étape ayant été décrite dans C. BlancheBenveniste et C. Jeanjean [1987].

Voyons à présent l'étude syntaxique menée sur ce corpus.

\section{Recensement et classement des CV : méthode utilisée}

42 Nous ne posons pas la phrase simple comme unité de description syntaxique; nous lui préférons la notion de construction verbale, qui se définit comme étant composée du verbe et des éléments qu'il régit. Cela nous permet, d'une part, de travailler uniquement sur les relations de dépendance qu'entretient le verbe avec les éléments qu'il régit, et d'autre part, de contourner la question polémique de la définition de la phrase ${ }^{6}$.

La linguistique descriptive, basée sur l'observation de corpus, ne peut pas être extensive ; un corpus, aussi important soit-il, est par nature restreint (il ne présente nécessairement pas toutes les possibilités de création syntaxique et lexicale).

La pronominalisation est un des outils principaux que nous utilisons. Elle consiste à appauvrir le lexique, afin de le catégoriser de manière systématique et non-équivoque, en ne retenant ainsi que le «squelette » de la construction verbale. Les proformes ${ }^{7}$ que nous avons utilisées sont en partie celles proposées par C. Blanche-Benveniste et al. dans Pronom et Syntaxe, auxquelles nous avons ajoutées celles que K. van den Eynde et P. Mertens ont proposées dans leur présentation du dictionnaire de valence Proton (Eynde et Mertens, $2001:$ p. 26).

\subsection{Constructions verbales à traiter}

\subsubsection{Recensement des constructions verbales : les verbes retenus}

La requête [\#v], qui permet d'obtenir toutes les formes verbales (infinitives, conjuguées, participiales) donne 15749 résultats, parmi lesquels on trouve des " déchets ", c'est-àdire des mots reconnus comme des formes verbales par Xcor20008, mais qui n'en sont pas: bouffe, communions, plus, etc. C'est un des problèmes que rencontrent les informaticiens-linguistes lorsqu'il s'agit de différencier deux formes homographes qui ne sont pas de même nature dans un corpus qui n'est pas étiqueté morpho-syntaxiquement. En étant très large, on peut compter $5 \%$ de « déchets ». Nous ne les avons pas comptés 
systématiquement, ce qui explique cette approximation. Le nombre total de constructions restantes approche donc 15000.

Une telle somme de matière première est difficilement traitable par une seule personne en un temps limité, quand bien même la méthode de traitement manuel est systématique (lorsque l'on a défini les propriétés de chaque type de constituant lexical et que l'on est certain, pour chacun d'entre eux, de pouvoir lui substituer une "proforme»). C'est pourquoi nous avons décidé de limiter le recensement des C.V. aux verbes les plus fréquents dans notre corpus, sans toutefois avoir fixé la limite fréquentielle, car le traitement des données, sur ce principe, se fait par tri décroissant (on étudie tout d'abord le verbe qui présente le plus grand nombre d'occurrences dans le corpus, puis celui qui lui succède immédiatement sur le même principe fréquentiel, etc.). En l'état actuel des travaux, nous ne pouvons donc pas affirmer clairement le nombre de verbes finalement étudiés. Notre objectif est d'arriver à traiter au moins la moitié des 15000 C.V. recensées.

\subsubsection{Les « transformations »}

Il y a deux manières de considérer la construction verbale suivante, qui comprend deux verbes recteurs, le deuxième entrant lui-même dans la rection du premier :

Ex : je pense que ça ça arrivera jamais (MADMO 34 12).

Le « verbe non régi » est ici pense. En revanche, arrivera est régi par pense.

- Soit on analyse cette construction comme un tout, en la pronominalisant en je le pense, tout en précisant la nature de l'élément lexical de départ que l'on retrouve ici sous le pronom le (Que-Phrase). C'est l'analyse la moins fine et la moins précise, qui aurait cependant l'avantage de réduire considérablement le corpus des constructions verbales à traiter dans le cadre de notre travail. Cette analyse ne prend donc en compte que les constructions des verbes non régis.

- Soit on l'analyse comme un ensemble de deux structures : les constructions verbales régies par un autre verbe peuvent toutes être réduites à des constructions non régies (la Quephrase de notre exemple peut ainsi être transformée et réduite à ça arrivera jamais, et étudiée comme telle). La deuxième analyse possible est donc celle qui consiste à détailler la proposition de départ et à la considérer comme un tout composé de deux ensembles, et donc de deux constructions à analyser : la première est la construction complète je le pense, la deuxième est le résultat de la transformation de la Que-phrase en construction non régie, ça arrivera jamais.

Nous avons retenu la méthode d'analyse la plus précise, qui consiste à retenir à la fois les verbes non régis (qui sont à l'origine de la construction verbale entière lorsqu'il y a plusieurs verbes recteurs) et régis (qui dépendent d'un autre verbe).

\subsubsection{Un exemple : le cas du verbe aller ${ }^{9}$}

Dans ce corpus, on trouve 428 verbes différents, possédant chacun une ou plusieurs constructions. Nous distinguons différents types de constructions en fonction des propriétés syntaxiques et distributionnelles des divers éléments construits par le verbe. Par exemple, le verbe aller possède, selon notre classement, six catégories principales de constructions ${ }^{10}$. Certaines de ces catégories sont elles-mêmes sous-catégorisées, comme nous allons le montrer, de manière à préciser un ou plusieurs de leurs traits. Par exemple, la catégorie notée 1-[abc], comprend, en 1-a, les constructions où l'on trouve le trait 
+personnel du sujet et un syntagme verbal à l'infinitif avec ses propres compléments ${ }^{11}$, en $1-b$, le trait -personnel du sujet et $\operatorname{SVinf} \Omega$, et en 1-c le trait -personnel du sujet et un Svinf.

Nous appelons +personnels les compléments dont le référent possède le trait « humain ». Nous le symbolisons par je, dans la mesure où le je est le pronom le moins ambigu: il correspond toujours à un individu dans une situation d'énonciation. Nous appelons personnels les compléments dont le référent est « inanimé »; nous le symbolisons par ça ou il.

Le chiffre ou le nombre apparaissant après l'indice de construction que nous venons d'expliciter est la fréquence d'emploi de la structure étudiée à l'intérieur de notre corpus. Ainsi [1a-165] signifie que la construction de type « je vais Svinf $\Omega$ » (pour laquelle aller est constructeur) apparaît 165 fois dans notre corpus. Nous allons à présent observer les différents types de constructions du verbe aller.

La première de ces catégories comprend les constructions dans lesquelles aller est suivi d'un syntagme verbal à l'infinitif accompagné ou non de complément(s) et qu'il est dans ce cas :

a) soit recteur, auquel cas aller accepte un pronom de type $y$ parfois ambigu (il est locatif, tout en reprenant le $\operatorname{SVinf} \Omega$, dans l'exemple suivant ${ }^{12}$ ), son sujet est toujours de type +personnel (cat. «1a» dans le tableau) :

Ex. 1 : tu vas faire des stages à Yssingeaux (СHOCM 10 9,7)

- j'y vais (là, pour ça)

b) soit il est utilisé avec un emploi modal, auquel cas la pronominalisation en y est impossible, comme le notait déjà Xiao-Quan Chu (1987 : p. 76). Le sujet de la CV peut être soit +personnel (type je, ex. 2), soit -personnel (type ça, ex. 3), et le Svinf peut être accompagné ou non de compléments (cat. 1a', $1 \mathrm{~b}$ et $1 \mathrm{c}$ dans le tableau). Dans ce cas, aller n'a pas de valence propre : c'est alors le verbe à l'infinitif qui est recteur de ces éléments. Deux tests permettent de repérer ce type de constructions (C. Blanche-Benveniste et al., 1990 : pp. 199-202) : si aller est en emploi modal (il va pleuvoir), il n'est pas conjugable au passé composé (*il est allé pleuvoir), et il n'y a pas de substitution possible sur son paradigme (*il court pleuvoir); s'il ne l'est pas, il accepte ces propriétés (il va faire ses courses > il est allé faire ses courses > il court faire ses courses).

Ex. 2 : le vendeur il va pouvoir aussi vous conseiller (HOTEV 26 12,16)

*j'y vais (pouvoir vous conseiller) (sujet +personnel)

*je suis allé pouvoir vous conseiller

*je cours pouvoir vous conseiller

Ex. 3 : j'espère que ça va durer enfin je sais pas (MADMO 34 4,6)

*ça y va (sujet -personnel)

*ça est allé durer

*ça court durer

En dépouillant notre corpus, nous avons remarqué une régularité intéressante dans la distribution du verbe aller: suivi d'un infinitif, il a toujours un emploi modal lorsque cet infinitif est lui-même un modal (pouvoir, vouloir...), un auxiliaire d'aspect (commencer, venir ...), un verbe construisant un adjectif ${ }^{13}$ (être, devenir...), le verbe avoir, ou enfin un verbe de déplacement (monter, partir, venir...). Notons ici que pour cette catégorie 1, lorsque le trait du sujet est [-personnel], aller est toujours dans un emploi modal, alors que ses emplois sont répartis lorsqu'il possède un sujet de type [+personnel]. 
Dart, il semblerait que dans le cas où aller est suivi d'un verbe à l'infinitif (exceptés ceux cités au paragraphe précédent) et d'un locatif, il soit toujours employé dans son sens " plein ", comme dans l'exemple déjà cité :

tu vas faire des stages à Yssingeaux $>$ tu $\mathbf{y}$ vas faire des stages

La présence contextuelle presque systématique d'un locatif dans ce type de constructions nous conduit à penser que le français parlé a tendance à désambiguïser ces relations de dépendance en « redonnant » au verbe aller son sens locatif. Cependant, pour ce type de constructions, deux interprétations sont toujours en concurrence : le locatif peut être dépendant soit du verbe aller, soit du verbe à l'infinitif :

tu vas faire des stages à Yssingeaux

tu y vas faire des stages (c'est dans ce cas le verbe aller qui régit le locatif).

tu vas y faire des stages (c'est ici le verbe faire qui régit le locatif).

Nous avons décidé d'inclure ce type de constructions (aller emploi modal + Vinf) sous l'entrée du verbe aller car il est manifestement courant en français parlé.

La deuxième catégorie comprend les constructions à l'intérieur desquelles aller régit un syntagme prépositionnel, et pour lesquelles il prend son sens "plein » de verbe locatif. Les 7 sous-catégories sont effectuées essentiellement en fonction de la présence ou non d'éléments régis se rajoutant à la structure de « base », qui est la suivante :

Ex. 4 : j'allais chez mon boucher (INSTI 28 3,13)

j'y allais

La troisième catégorie est composée :

- des constructions dans lesquelles aller régit soit seulement un sujet de type ça (cat. 3a) :

- Ex 5 : les sorbets ça va c'est quelque chose de relativement simple CHOCM 10 12,12

- des constructions dans lesquelles le verbe aller régit un élément de type ça et un élément de type ainsi (cat. 3b) :

- Ex. 6: le soixante-dix pour euh enrober ça va très bien parce que c'est très liquide CHOCM 10 16,13

- ça va ainsi

La quatrième catégorie comprend les constructions dans lesquelles aller régit un sujet de type ça, un élément de type te, et un élément de type ainsi (cat. 4);

Ex. 8 : qu'on leur disent ben vous êtes belles ça vous va bien vous êtes euh ça elles aiment les clientes FANMO 16 20,8

ça vous va ainsi

61 La cinquième catégorie est composée de constructions de type s'en aller, qui semblent figées (cat. 5). Le sujet est de type +/- personnel :

Ex. 9 : j'y vais je prends et je m'en vais (COMMO 14 4,3)

je m'en vais

Ex. 10 : la rue qui s'en va au théâtre la rue Bréchimbault (MALLM 36 9,2)

Elle (-pers) s'en va

Enfin, la sixième catégorie comprend les constructions dans lesquelles aller ne régit que son sujet, et dans lesquelles il est toujours à l'impératif (cat. 5) :

Ex. 11 : comment ça s'appelle les Bootlegers les mmh allons bon Claude Luter Maxime Sauri La Ferrière (MUSIK 46 7,5) 


\subsubsection{Présentation du tableau} solidaires sont celles de la réalisation du syntagme prépositionnel pronominalisé en où. On observe par exemple que pour le deuxième type de constructions ( 2 [ $\mathrm{a} \mathrm{b} \mathrm{c} \mathrm{d} \mathrm{e} \mathrm{f} \mathrm{g]),} \mathrm{les}$ quatre prépositions [à, en, dans, chez] peuvent apparaitre dans le corpus en tête de ce Sprep.

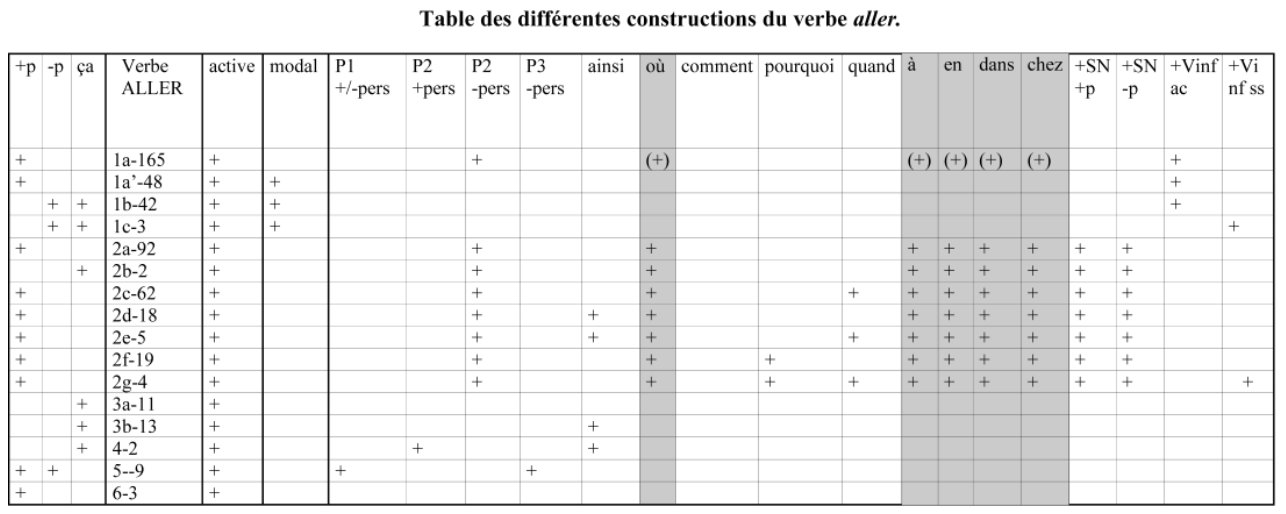

Pour une aide visuelle à la lecture de ce tableau, voici pour chaque construction un exemple tiré du corpus :

1-a Je vais vinf $\Omega$ (+/- élément locatif) (verbe « plein »)

CHOCM 10 9,7 mais bon sinon tu vas faire des stages à Yssingeaux

1-a' Je vais $\operatorname{vinf} \Omega$ (emploi modal)

HOTEV 26 12,16 le vendeur il va pouvoir aussi vous conseiller

1-b ça va $\operatorname{vinf} \Omega$ (emploi modal)

MUMON 44 11,12 enfin quand je vais rentrer ça va être nickel quoi donc voilà

1c-ça va vinf (emploi modal)

MADMO 34 4,6 j'espère que ça va durer enfin je sais pas

2-a j'y vais, là

INSTI 28 3,13 j'allais chez mon boucher il savait ce que je prenais

2-b ça y va, là

MUSIK 46 17,5 à l'occasion mais ça allait pas bien plus loin

2-c j'y vais quand

MADMO 34 9,12 l'exposition des guitares j'y allais presque tous les enfin pour dire

tous les jours

2-d j'y vais ainsi

BRUNMO 813,6 on y est = allé tous les quatre et euh on a gagné les élections

2-e j'y vais quand ainsi

MAMMO 38 8,13 elle a gagné le voyage mais quand tu vas en voyage c'est avec

d'autres directeurs 
2-f j'y vais pourquoi

COIFM 12 9,14 ils vont pas chez les autres pour se présenter

2-g j'y vais quand pourquoi

COMMO 14 3, j'y vais tous les lundis parce que ben la semaine XXX je prends je travaille de neuf heures à dix-neuf heures

3-a Ça va

CHOCM 10 12,12 les sorbets ça va c'est quelque chose de relativement simple

$3 \mathrm{~b}$ Ça (ou P0-pers) va ainsi

CHOCM 10 16,13 le soixante-dix pour euh enrober ça va très bien parce que c'est

très liquide

4- Ça te va ainsi

FANMO 16 20,8 qu'on leur disent ben vous êtes belles ça vous va bien vous êtes euh

ça elles aiment les clientes

5- je m'en vais / Po -pers s'en va

- Je m'en vais :

COMMO 14 4,3 euh j'y vais je prends et je m'en vais et en fait je vais toujours dans

la même grande surface

- Elle (-pers) s'en va :

MALLM 36 9,2 la rue qui s'en va au théâtre la rue Bréchimbault

6- allez

MUSIK 46 7,5 comment ça s'appelle les Bootlegers les mmh allons bon Claude Luter

Maxime Sauri La Ferrière

\section{Conclusion et perspectives}

Un des problèmes posés par le classement d'objets, peu importe leur nature, est celui du choix de leurs propriétés distinctives. Sont-elles suffisamment pertinentes pour être retenues? Leur choix est-il opératoire? Ce n'est qu'après avoir dépouillé et analysé un certain nombre de constructions que l'on peut apprécier la valeur des propriétés en question. La taxonomie implique souvent des tâtonnements méthodologiques, qui sont d'autant plus paralysants que le nombre des données à traiter est important.

Par exemple, le cas du verbe aller est révélateur: nous avons trouvé deux types de constructions apparemment très proches, à l'intérieur desquelles aller est présent, mais dans un emploi différent dans les deux cas (dans la $1^{\text {ère }}$ catégorie, a et a'). Ces deux types de constructions utilisent un syntagme verbal à l'infinitif accompagné ou non de complément(s) et un sujet de type +personnel. Ce qui nous a permis dans un premier temps de classer aller soit dans un emploi modal, soit dans un emploi "plein » est essentiellement le fait qu'il puisse être précédé ou non d'un pronom de type $y$ (qu'il accepte lorsqu'il n'est pas modal), et qu'il puisse être ou non conjugué à un autre temps que le présent ou l'imparfait de l'indicatif. Ces tests sont connus et utilisés depuis longtemps, et, en cela, cette étude n'apporte rien de nouveau. Cependant, une fois le classement effectué à partir de ces tests, une observation approfondie des exemples du corpus nous a permis de montrer que dans son sens plein, pour cette catégorie 1 , aller est très souvent accompagné dans un contexte plus ou moins large d'un élément locatif. Nous pensons qu'il s'agit ici d'une stratégie de désambiguïsation assez caractéristique de l'oral. En effet, lorsque aller (suivi de $\operatorname{Svinf}(\Omega / \varnothing)$ ) régit un élément locatif, il perd toujours son trait modal, autrement dit, il est toujours employé comme verbe constructeur, avec son sens plein de déplacement. En outre, nous avons vu qu'il régit dans ce cas toujours un sujet +personnel. 

En outre, la possibilité d'effectuer un classement fréquentiel des différents types de constructions permettrait à l'enseignant d'avoir un programme d'apprentissage évolutif, en proposant tout d'abord à ses apprenants les constructions les plus fréquemment utilisées, puis, au fur et à mesure de leurs progrès, celles dont la fréquence est moindre.

En TALN, l'heure est actuellement au travail sur les grands corpus de données attestées, qu'il s'agisse de textes écrits ou de transcriptions d'oral. Les corpus oraux posent de nombreux problèmes aux informaticiens, notamment l'absence de ponctuation, mais des recherches sont en cours pour formaliser la syntaxe du français parlé ( $C f$. p.ex. Christophe Benzitoun, DELIC, 2003: "Un langage "pivot» pour articuler description et formalisation: l'exemple des verbes introducteurs de Que-Phrases", Batz-sur-Mer, Colloque Récital.). Cette étude pourrait donc apporter des renseignements utiles sur le fonctionnement et la distribution des verbes à l'oral, et ainsi faciliter la formalisation des données pour le traitement automatique du langage.

\section{BIBLIOGRAPHIE}

BIGGS, P. et BLANC, M., 1971, «L'enquête sociolinguistique sur le français parlé à Orléans ", in Le français dans le monde, $\mathrm{n}^{\circ} 85$, décembre 1971, pp. 16-25

BLANCHE-BENVENISTE C., BILGER M., ROUGET C., VAN DEN EYNDE K., 1990, Le Français parlé, études grammaticales, Paris, éd. du C.N.R.S.

BLANCHE-BENVENISTE C., JEANJEAN C., 1987, Le Français parlé, transcription et édition, Paris, INALF, Didier Érudition

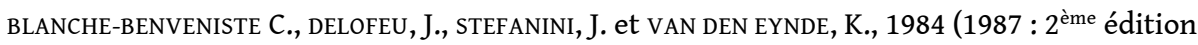
augmentée), Pronom et syntaxe, l'approche pronominale et son application au français, Paris, Selaf CÉLÉRIER, P., MAILLARD, J.-P., 1979, Dictionnaire des Structures Fondamentales du français (D.S.F.), Paris, CLE International

CHU, X.-Q., 1989, «Examen de la structure des enchaînements de verbes modaux », Recherches sur le français parlé n 9, Aix, Publications de l'Université de Provence, pp. 75-86

DELIAS-ROUSSARIE, E., et DURAND, J. (éds.), 2003, Corpus et variations en phonologie du français, Toulouse, Presses Universitaires du Mirail, coll. « Interlangues linguistique et didactique » EYNDE, K. van den \& MERTENS, P. (2001) La Syntaxe du verbe, l'approche pronominale et le lexique de valence PROTON, Preprint $n^{\circ} 174$, Departement of Linguistics, K.U.Leuven, pp. 36 (PDF File) (published in modified form in French Language Studies 13, 2003)

FREYSSINET-DOMINJON, J., 1997, Méthodes de recherche en sciences sociales, Paris, Montchrestien, Coll. «AES »

Corela, HS-1 | 2005 
GADET F., 1971, « Recherches récentes sur les variations sociales de la langue », Langue Française, $\mathrm{n}$

• 9, Linguistique et société, Paris, Larousse, pp. 74-81

GIRON, S., 2001, Corpus Allier, transcription de 7 h30 de français parlé dans l'Allier (03) en zones urbaines

GREIDANUS, T., 1990, Les constructions verbales en français parlé. Etude quantitative et descriptive de la syntaxe des 250 verbes les plus fréquents, Tübingen, Niemeyer, coll. « Linguistiche Arbeiten »

GRoss, M., 1975, Méthodes en syntaxe, Régime des constructions complétives, Paris, Hermann

GROSS, M., 1986 (2 ème éd.), Grammaire transformationnelle du français, vol. 1, Syntaxe du verbe, Malakoff, Cantilène, Coll. « Systématique de la langue française »

LE GOFFIC, P., COMBE MCBRIDE, N., 1975, Les constructions fondamentales du français, Paris, Hachette/

Larousse, Coll. « Le français dans le monde-B.E.L.C. »

WILLEMS, D., 1981, Syntaxe, lexique et sémantique. Les constructions verbales, Gand, Publications de la

Faculté des Lettres de Gand

WILLEMS, D., 2001, «Classes et relations verbales. À la recherche d'une typologie intégrée », in

Travaux Linguistiques du Cerlico ${ }^{\circ} 15$ (Morphosyntaxe du lexique, Catégorisation et mise en

discours), Presses Universitaires de Rennes, pp. 285-302

\section{NOTES}

1. Nous tenons ici à remercier Geoffrey Williams et les PUR, qui nous ont aimablement autorisée à reproduire en partie cette présentation, que nous avions déjà faite lors des $3^{\text {èmes }}$ Journées de Linguistique de Corpus (Lorient, 2003), et dont les Actes sont à paraître.

2. «L'équipe DELIC (Jeune Equipe JE 2328) s'intéresse à la description des structures morphosyntaxiques et lexicales en français, en synchronie comme en diachronie. Sa méthodologie repose sur l'utilisation systématique de grands corpus oraux et écrits, à l'aide d'outils informatiques appropriés (concordanciers, étiqueteurs, outils de gestion et de navigation, etc.).» cf. http://www.up.univ-mrs.fr/delic/index.html. Les transcriptions sont effectuées en orthographe normée ; pour plus de détails, cf. le site de DELIC, indiqué ci-dessus. DELIC, 2002, Projet « Corpus français parlé de référence », rapport final, non-publié.

3. C'est à ce niveau là que nous ferons référence si rien n'est indiqué ; sinon, nous signalerons le sous-type en note.

4. : http://p2pfr.free.fr/win/convertisseur/MPEGSuite.exe

5. : ( ) Microsoft Corporation, juin 1999.

6. Sur ce point, voir l'article d'Alain Berrendonner, «Les deux syntaxes », in Verbum, 2002, tome XXIV $n^{\circ} 1-2$, Presses Universitaires de Nancy, et celui de Claire Blanche-Benveniste, «Phrase et construction verbale ", dans la même revue.

7. Nous préférons ce terme à celui de pronom, dont la définition s'avère problématique comme le notaient déjà $\mathrm{F}$. Brunot et $\mathrm{E}$. Benveniste.

8. Nous tenons ici à remercier Jean-Pierre Adam, qui nous a autorisée à utiliser le concordancier qu'il a conçu.

9. Nous tenons ici à remercier Mylène Blasco-Dulbecco qui nous a patiemment conseillée lors de la réalisation de cette partie. Si des erreurs ou des imperfections subsistent, elle n'en est cependant aucunement responsable.

10. cf. tableau page 47 . 
11. Que nous noterons désormais $\operatorname{Svinf} \Omega$ (avec complément(s)) ou Svinf (sans complément), selon la notation de Gross [1975].

12. Tous les exemples attestés de cette catégorie ne comprennent pas nécessairement de locatif régi par aller, c'est pourquoi le complément de type où apparaît entre parenthèses dans le tableau p. 47 , comme substitution possible de $y$. Sans locatif, le Svinf $\Omega / \varnothing$ se pronominalise en $y$ : je vais faire mon stage > j'y vais, d'où l'ambiguïté relative à ce pronom lorsqu'un élément locatif est présent.

13. Verbes que la grammaire traditionnelle nomme "verbes d'état »

14. : Traduction Automatique du Langage Naturel.

\section{RÉSUMÉS}

Nous proposons de nous intéresser à une méthode de classement des constructions verbales relevées dans un corpus de français parlé (environ 15000 constructions inventoriées). Cette méthode présente quelques avantages : une représentation en tableau permet d'avoir une vision synthétique des types de constructions relevés dans notre corpus et de les classer selon la forme du verbe et les traits syntactico-sémantiques des sujets et compléments qu'il régit. En outre, le résultat de ce travail pourrait servir de base à un essai de typologie verbale, effectué en fonction de la complémentation des verbes étudiés. Nous présenterons en détail la méthode utilisée pour ce travail depuis la constitution du corpus (enquêtes de terrain et transcriptions), jusqu'à l'inventaire et au classement des constructions verbales.

We put forward a method of classification of verbal constructions coming from a French spoken corpus (about 15,000 listed constructions). The advantages of this method are the following: a representation in a table permits to have a synthetic vision of the types of constructions found in our corpus and to classify them according to the form of the verb and the syntactico-semantic features of the subjects and the complements which it governs. Moreover, the result of this work could be used as a basis for a verbal typology, carried out according to the complementation of the studied verbs. We will present in detail the method used for this work from the constitution of the corpus (investigations and transcriptions), to the inventory and the classification of verbal constructions.

\section{INDEX}

Mots-clés : constructions verbales, syntaxe, français parlé, corpus

\section{AUTEUR}

\section{STÉPHANIE GIRON}

Université Blaise Pascal, Clermont-Ferrand II, Université Lumière, Lyon II (ATER) 